\title{
LA-UR- $97-3894$
}

Title:

OPTIMAL DETECTION OF NEAR-EARTH OBJECTS

Author(s):

Submitted to:

\section{Gregory H. Canavan, P-DO}

\section{RECEIVED \\ DEC 161997 \\ OSTI}

For Air Force Phillips Laboratory Meeting on

Space Debris Detection

Date: September 1997

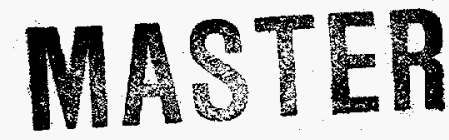

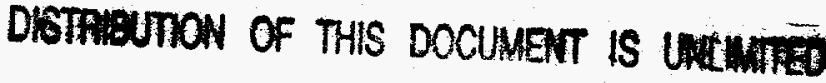


Los Alamos National Laboratory, an affirmative action/equal opportunity employer, is operaled by the University of California for the U.S. Department of Energy under contract W-7405-ENG-36. By acceptance of this article, the publisher recognizes that the U.S. Government retains a nonexclusive, royalty-free license to publish or reproduce the published form of this contribution, or to allow others to do so, for U.S. Government purposes. The Los Alamos National Laboratory requests that the publisher identify this article as work performed under the auspices of the U.S. Department of Energy. 


\section{DISCLAIMER}

This report was prepared as an account of work sponsored by an agency of the United States Government. Neither the United States Government nor any agency thereof, nor any of their employees, makes any warranty, express or implied, or assumes any legal liability or responsibility for the accuracy, completeness, or usefulness of any information, apparatus, product, or process disclosed, or represents that its use would not infringe privately owned rights. Reference herein to any specific commercial product, process, or service by trade name, trademark, manufacturer, or otherwise does not necessarily constitute or imply its endorsement, recommendation, or favoring by the United States Government or any agency thereof. The views and opinions of authors expressed herein do not necessarily state or reflect those of the United States Government or any agency thereof. 


\section{DISCLAMVER}

Portions of this docement may be illegible in electronic image products. Impges are produced from the best available original docement. 


\title{
OPTIMAL DETECTION OF NEAR-EARTH OBJECTS
}

\author{
Gregory H. Canavan
}

Improved technology and search strategies could significantly increase debris detection rates and tracking tracking accuracies with modest investment. Improving the focal planes of current telescopes could significantly increase detection rates. For large systems, proportional increases in aperture area and array sizes are appropriate.

The detection of space objects has shifted from long-exposure, distant searches ${ }^{1}$ to rapid searches of the whole sky made possible by improved detector and electronic technologies. ${ }^{2}$ Such approaches are also applicable to search and track of debris closer to the Earth. This note discusses improvements in ground-and space-based search sensors and search strategies that could make rapid search possible.

Optimal detection uses exposure times shorter than those typically used for detection at maximum range and scans the whole sky rapidly to maximize detection opportunities. The new element is technology. Few million detector focal planes and electronics make it possible for telescopes $\sim 1$ meter in diameter to produce adequate detection rates for objects with diameters ranging from centimeters at low Earth orbit (LEO) and meters at geosynchronous orbit (GEO) to kilometers at a few astronomical units. ${ }^{3}$

As array sizes are increased, the detection rate increases as the square root of the number of detectors; however, very large focal planes would eventually fill the telescope's flat field. To avoid overfilling, it would thereafter be necessary to increase aperture and detector count. Large ground-based systems have strongly diminishing marginal returns due to limitations from atmospheric turbulence, which could be relieved by smaller sensors in space.

Detection requirement. Low signal-to-noise ratio (SNR) detection necessitates long integration time, $t$. The signal power, $S_{r e q}$, required to achieve $S N R \approx 4-8$ is approximately

$$
\mathrm{S}_{\mathrm{req}}=\sqrt{ }\left(\mathrm{SNR}^{2} \mathrm{~B} / \mathrm{Qt}\right) \text {, }
$$

where $Q$ is the sensor quantum efficiency, and B is the background noise. Since $Q$ and $S N R$ are relatively fixed, $S_{\text {req }}$ scales roughly as $\sim \sqrt{ }(B / t)$.

Background noise in ground-based systems is $\mathrm{B} \approx \mathrm{B}$ " $\mathrm{A} \theta^{2}$, which is proportional to the telescope primary area $\mathrm{A}$ and angular pixel size $\theta . \mathrm{B} " \approx 0.63 / \mathrm{m}^{2}$-arcsec ${ }^{2}$. Atmospheric seeing limits $\theta$ to a few arcsec, so $B \sim A$, and $S_{r e q} \sim \sqrt{ }(A / t)$. 
The signal received by a sensor of aperture A from an object of diameter $\mathrm{D}$ at range $\mathrm{r}$ with solar illumination times albedo $\mathrm{J}$ is

$$
\mathrm{S}_{\mathrm{rec}} \approx \mathrm{JAD}^{2} / \mathrm{r}^{2} \text {, }
$$

where $A, D$, and $r$ of interest each vary over several orders of magnitude.

Sensitivity. Equating $S_{r e q}$ to $S_{r e c}$ determines the minimum detectable diameter

$$
\mathrm{D}=\mathrm{r}\left(\mathrm{SNR}^{2} \mathrm{~B}^{\prime \prime} \theta^{2} / \mathrm{J}^{2} \mathrm{QAt}\right)^{1 / 4}
$$

which scales as the range and inversely with the fourth root of the area-exposure product At. Figure 1 shows $\mathrm{D}$ as a function of $\mathrm{t}$ for $\mathrm{r}=40,000 \mathrm{~km}$, i.e., geosynchronous orbit (GSO). The top curve is for a $0.1 \mathrm{~m}$ primary mirror. It can detect $\sim 0.1 \mathrm{~m}$ objects with $10 \%$ reflectivity with $0.1 \mathrm{~s}$ exposures. With $1 \mathrm{~s}$ it could detect an object about half that size. The bottom curve is for a $1 \mathrm{~m}$ primary, which could detect $\mathrm{a} \sim 4 \mathrm{~cm}$ object in $1 \mathrm{~s}$, in accord with the $1 / \mathrm{A}^{1 / 4} \sim 1 / 100^{1 / 4} \sim 1 / 3$ scaling of Eq. (3).

Debris. Near-Earth space contains debris with a distribution

$$
\mathrm{N} \approx \mathrm{K} / \mathrm{D}^{\mathrm{n}} \approx \mathrm{K}_{\mathrm{O}} \mathrm{e}^{-\mathrm{kr}} / \mathrm{D}^{\mathrm{n}} \text {, }
$$

where $\mathrm{k}$ represents the decrease of the overall debris density with altitude, and $\mathbf{n}$ is a constant that characterizes the debris distribution over object size at each altitude. A sensor with FOV w searches solid angle at rate $w / t$. If it can detect objects to range $r$, each exposure searches a volume $\sim \mathrm{w} \mathrm{r}^{2} \mathrm{dr}$. Thus, its volumetric search rate is

$$
\begin{aligned}
\mathrm{R} & \approx(w / t) \int \mathrm{dr} \mathrm{r}^{2} \mathrm{~N}=(\mathrm{w} / \mathrm{t}) \int \mathrm{dr} \mathrm{r}^{2} \mathrm{~K} / \mathrm{D}^{\mathrm{n}} \\
& \approx(\mathrm{w} / \mathrm{t}) \sqrt{ }\left(\mathrm{J}^{2} \mathrm{QAt} / \mathrm{SNR}^{2} \mathrm{~B}^{\prime \prime} \theta^{2}\right) \int \mathrm{dr} \mathrm{r}^{2} \mathrm{~K}_{\mathrm{O}} \mathrm{e}^{-\mathrm{kr} / \mathrm{r}^{2}} \\
& \approx(\mathrm{w} / \mathrm{t}) \sqrt{ }\left(\mathrm{J}^{2} \mathrm{QAt} / \mathrm{SNR}^{2} \mathrm{~B}^{\prime \prime} \theta^{2}\right) \mathrm{K}_{0} / \mathrm{k},
\end{aligned}
$$

where the first line substitutes from Eq. (4); the second line uses Eq. (3) for D and assumes $n \approx 2$, which is as accurate as the data for $1-10 \mathrm{~cm}$ debris; ${ }^{4}$ and the third line performs the integral over all space using $\mathrm{k} \approx 1 / 1,000 \mathrm{~km}$. The extension to all space is acceptable in that the result is insensitive to the lower limit, and hence to the minimum object size. The use of this value of $\mathrm{k}$ is an adequate average for the first few tens of thousands of kilometers of range. ${ }^{5}$

$\mathrm{K}_{\mathrm{O}}$ is determined by integrating $\int \mathrm{dr} \mathrm{r}^{2} \mathrm{~N}$ over all space and setting it equal to the number of particles larger than $\sim 1 \mathrm{~cm}$. There are $\sim 8,000$ pieces of debris larger than $\sim 20-30 \mathrm{~cm}$ in the AF Space Command Catalog, which with the scaling above implies $\sim 10^{5}$ particles larger than $1 \mathrm{~cm}$, which gives $\mathrm{K}_{\mathrm{O}} / \mathrm{k} \sim 10^{-18} / \mathrm{km} / 10^{-3} / \mathrm{km} \sim 10^{-15} / \mathrm{km}$.

Figure 2 shows $\mathrm{R}$ as a function of $\mathrm{t}$ for $0.1,0.3$, and $1 \mathrm{~m}$ telescopes. The top curve is for 
$1 \mathrm{~m}$. It falls from about 300 detections $/ \mathrm{s}$ at $\mathrm{t}=0.01 \mathrm{~s}$ to $10 / \mathrm{s}$ at $10 \mathrm{~s}$, i.e., the detection rate falls inversely as the square root of $\mathrm{t}$. The curve for $0.3 \mathrm{~m}$ is a factor of $\sim 3$ below it, it accord with the

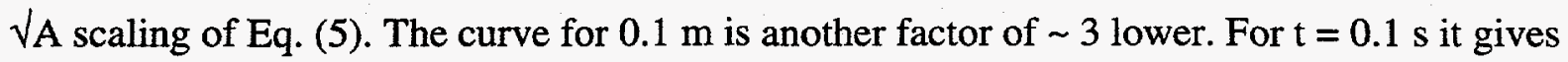
about 10 detections per second.

High search rate sensors. For a sensor to cover the whole fresh sky each month requires a search rate $\approx 100 \mathrm{deg}^{2} / \mathrm{hr}$. With a $\sim 1 \mathrm{deg}^{2} \mathrm{FOV}$, that would require $\sim 100$ exposures $/ \mathrm{hr}$, which would permit $\sim 30 \mathrm{~s}$ per exposure, or an actual exposure time of about $10 \mathrm{~s}$. To search the sky each night in the terminator would require a rate 10-100 times larger, or an exposure time of 1$0.1 \mathrm{~s}$. To achieve a search rate $W^{\prime}$ with a sensor FOV $w$ requires an exposure time $t \approx w / W^{\prime}$. Shorter exposure times would cover parts of the sky twice without producing additional discoveries; longer exposures would leave parts of the sky uncovered. Thus, $t \approx w / W^{\prime}$ is the optimum time for maximizing the rate of detection, although it is generally shorter than the time for maximizing the range of detection, the usual metric. Since B", and $\theta, S N R$, and $Q$ are relatively fixed, for search rate $W^{\prime}, \mathrm{R}$ scales as

$$
R_{\text {opt }} \sim(w / t) \sqrt{ }(A t) \sim W^{\prime} \sqrt{ }\left(A w / W^{\prime}\right) \sim \sqrt{ }\left(A w W^{\prime}\right),
$$

which apparently depends on $A, w$, and $W^{\prime}$. The actual scaling of $R_{o p t}$ is simpler.

Detector scaling. A sensor with f-number (focal length / primary diameter) $F$ and focal plane array with $\mathrm{N}$ detectors of pitch $\mathrm{d}$ has a geometric FOV

$$
\mathrm{w} \approx \mathrm{N}(\mathrm{d} / \mathrm{F})^{2 / A} \text {, }
$$

so its optimized search rate scales as

$$
R_{\text {opt }} \sim \sqrt{ }\left(A w W^{\prime}\right) \sim \sqrt{ }\left(A\left[N(d / F)^{2} / A\right] W^{\prime}\right) \sim(d / F) \sqrt{N} .
$$

A cancels out, so $R_{\text {opt }}$ only depends on $d / F$ and $N$. F cannot be decreased much below $F \approx 2$ without degrading optical quality, and $\mathrm{d}$ changes only with detector development, so increasing the number of detectors has the greatest potential for increasing detection rates.

$R_{o p t}$ is independent of $A$ because the light added by increasing $A$ is just offset by the reduction of FOV it produces. However, increasing A would just provide more flat region in the focal plane for additional detectors, if needed. If space is available in the focal planes of existing telescopes, the most effective way to improve them is to improve their focal planes.

Very large focal planes. For given $\mathrm{A}$, as $\mathrm{N}$ increases, w increases as N/A in accord with Eq. (7). At some value of $\mathrm{N}$, the maximum usable field of view, $w_{\max }$, will be reached. Further increases in $\mathrm{N}$ would put the added detectors outside of the region of acceptable focus. Thereafter, A must be increased in proportion to $\mathrm{N}$ to maintain image quality, and $\mathrm{R}_{\mathrm{Opt}}$ $\sqrt{ }\left(A w_{\max }\right) \sim \sqrt{ } \mathrm{A}$, in which aperture again has utility. The transition between the two limits occurs where $w_{\max } \approx N(d / F)^{2 / A}$, or 


$$
\mathrm{N} / \mathrm{A}=\mathrm{w}_{\max }(\mathrm{F} / \mathrm{d})^{2},
$$

which indicates that for a given class of optical designs, i.e., $w_{\max }$ and F/d, $N$ and $A$ should be increased proportionally.

For nominal parameters this transition occurs at N/A $\approx(1 \mathrm{deg} / 57.3 \mathrm{deg} / \mathrm{rad})^{2}(2 / 25$ micron) $)^{2} \approx 4 \times 10^{6} / \mathrm{m}^{2}$. For $\mathrm{N}$ smaller sensors should follow $\sqrt{\mathrm{N}}$ scaling; for larger arrays, they should follow $\sqrt{ }$ A scaling. As detector technology improves and $d$ decreases, the transitional value of N/A will increase as $1 / \mathrm{d}^{2}$. When the sensor is designed against the limit of Eq. (9)

$$
R_{\text {opt }} \sim\left(w_{\max } A N(d / F)^{2}\right)^{1 / 4} \sim(A N)^{1 / 4},
$$

which uses the benefits of both $\mathrm{N}$ and $\mathrm{A}$ to improve detection.

Cost scaling. $R_{\text {opt }}$ initially increases as $\sim \sqrt{ }$ N. Sensor costs are roughly linear in $N$ and A. Thus, either maximizing $R$ while holding cost fixed or minimizing cost while holding $R$ rate fixed sees no benefit from $A$ to offset its cost until the limit on focal plane area is reached. For that reason, optimal ground-based search systems tend to have large focal planes with apertures just large enough to contain them, which balances search rate and range to maximize detection rate. That is a different balance that that struck in earlier search designs, which had large telescopes, small focal planes, and long integration to maximize information per exposure.

The return per unit investment falls as $1 / \sqrt{ } \mathrm{N}$ or $1 / \sqrt{ } \mathrm{A}$, which means that even properly optimized ground-based systems have strongly diminishing marginal returns. Tracing back through the analysis above shows that these limitations stem from the inability of ground-based sensors to achieve pixel sizes smaller than those dictated by atmospheric turbulence. That limitation could be overcome by space-based systems, which are discussed elsewhere. ${ }^{6}$

Summary and conclusions. Technologies and strategies for space search and detection have undergone significant changes. Earlier work concentrated on deep searches with long exposures. Improvements in detectors and electronics for ground-based search sensors make faster search possible and more efficient. Optimal searches use exposure times proportional to sensor FOV, which are much shorter than those typically used for detection at maximum range. The search, signal and noise for such processes can be modeled analytically and combined into parameter combinations of adequate sensitivity. Space debris can be modeled to the accuracy required, and performance assessment can be evaluated analytically for distributions of interest. The resulting detection rate is a bilateral function of the search rate and the product of sensor area and exposure time, which falls from a few hundred per second to a few tens per second as exposure time increases and aperture diameter falls.

In improving current telescopes, area cancels out, and the rate only depends on the number of detectors, so increasing the number of detectors has the greatest potential for increasing detection rates in the near term. However, as the array size increases, FOV increases, 
so at some value, the maximum usable field of view is be reached. Further increases in detectors would put them outside of the region of acceptable focus, so aperture must be increased in proportion to detectors to maintain performance.

Maximizing rate or minimizing cost shows no benefit from aperture until the limit on focal plane area is reached. Thus, optimal ground-based search systems tend to have large focal planes with apertures just large enough to contain them, which balances search rate and range to maximize detection rate. Even properly optimized ground-based systems have strongly diminishing marginal returns due to atmospheric turbulence, which could be overcome with smaller space-based sensors. 


\section{References}

${ }^{1}$ E. Shoemaker, Report of the Near-Earth Object Survey Working Group (Washington, DC, NASA Hq., June 1995).

${ }^{2}$ G. Canavan, "Cost and Benefit of Near-Earth Object Detection and Interception," T. Gehrels, ed., Hazards Due to Comets \& Asteroids (Tucson, University of Arizona Press, 1994).

${ }^{3}$ G. Canavan, "Optimal Detection of Near Earth Object Threats," J. Remo, ed, UN International Conference on Near-Earth Objects, Annals of the New York Academy of Sciences, vol. 822, 30 May 1997, pp. 539-543.

${ }^{4}$ G. Gleghorn, Orbital Debris (Washington DC, national Academy of Science, National Research Council, 1995), p. 80, Fig. 4-1.

${ }^{5}$ G. Gleghorn, Orbital Debris ,op. cit., p. 66, Fig. 3-3.

${ }^{6}$ G. Canavan, "Optimal Detection of Short-Warning Near-Earth Object Threats," Proceedings Space 96, Fifth International Conference on Space, Albuquerque, NM, June 1996. 
:०-
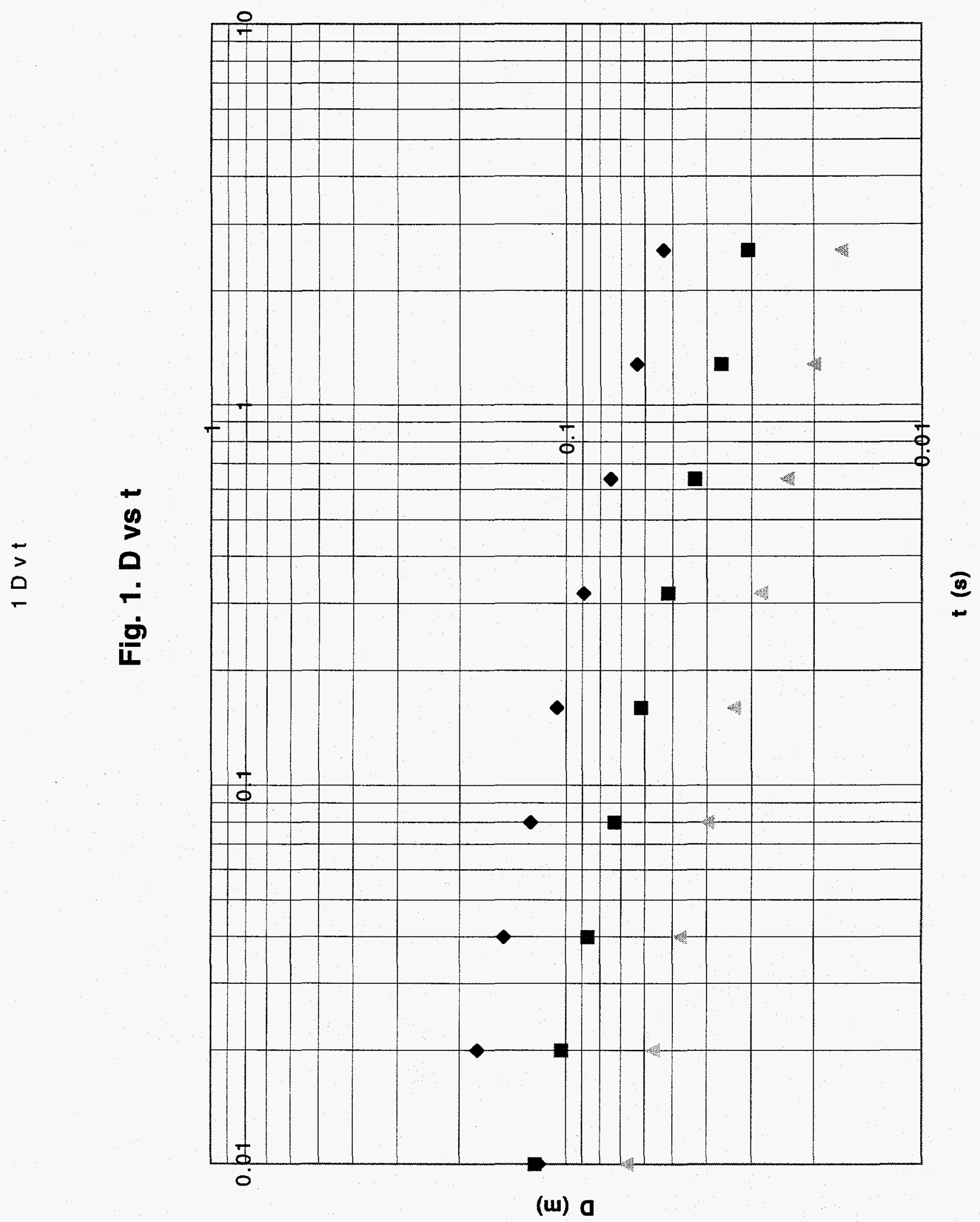


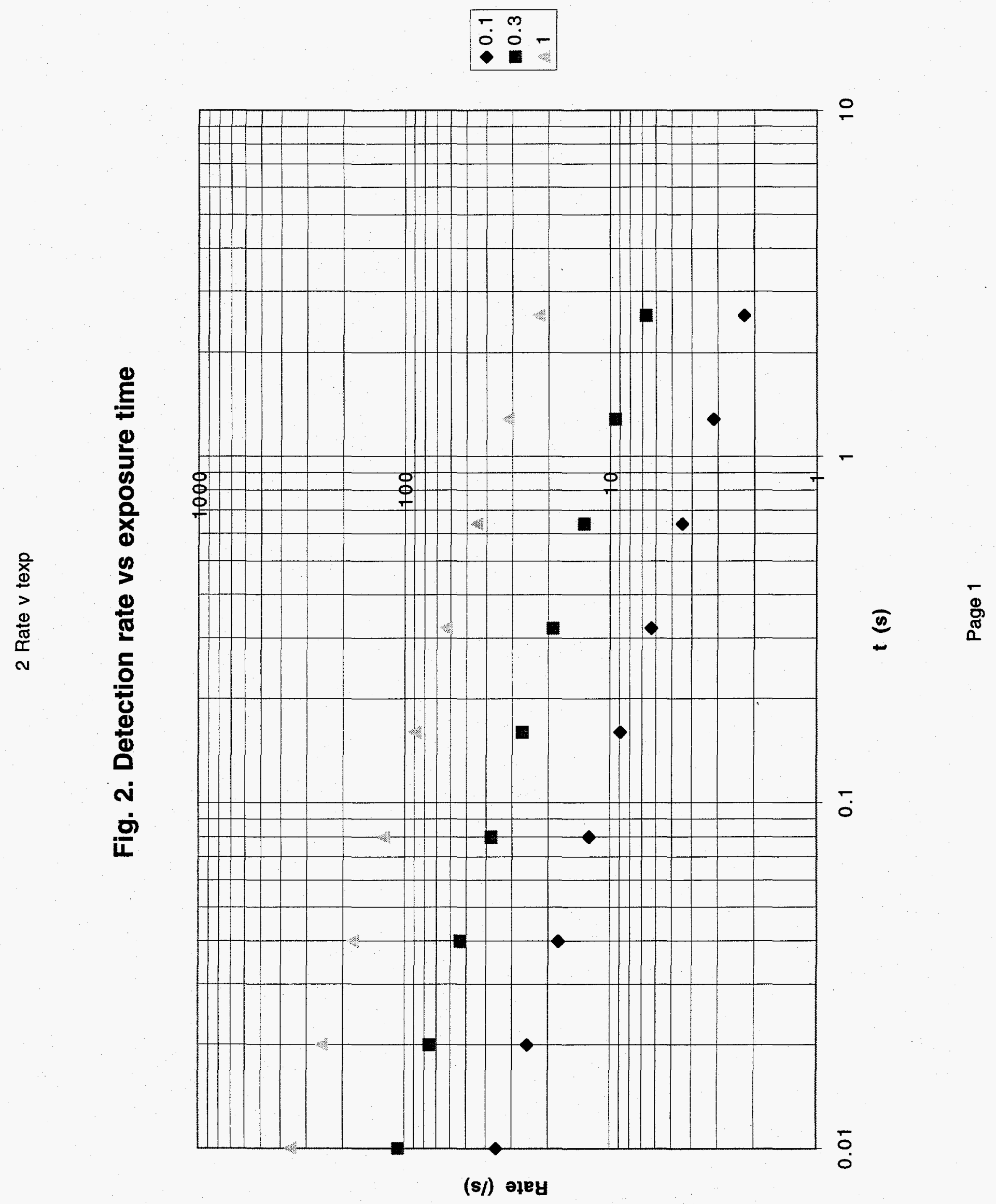

